\title{
Origins of Solvation Forces in Confined Films
}

\author{
Jianping Gao, W. D. Luedtke, and Uzi Landman* \\ School of Physics, Georgia Institute of Technology, Atlanta, Georgia 30332
}

Received: January 27, 1997; In Final Form: March 17, $1997^{\otimes}$

\begin{abstract}
Comparative investigations of equilibrium structures, solvation forces, and conformational dynamics of thin confined films of spherical molecules, straight-chain alkanes ( $n$-hexadecane and $n$-tetracosane), and a branched alkane (squalane) are performed using a newly developed grand canonical ensemble molecular dynamics method for simulations of confined liquids. The method combines constant pressure simulations with a computational cell containing solid surfaces and both bulk and confined liquid regions in equilibrium with each other. For all the molecular liquid systems layered density oscillations in the confined films are found for various widths of the confining gap. The solvation force oscillations as a function of the gap width for the spherical and straight-chain alkane liquids are more pronounced, exhibiting attractive and repulsive regions, while for the branched alkane the solvation forces are mostly repulsive, with the development of shallow local attractive regions for small values of the gap width. Furthermore, the calculated free-energy changes upon increased confinement of these systems show oscillatory attractive values for the spherical and straightchain molecular films and overall repulsive (positive) values for squalane. The energetic and entropic components of the solvation forces are investigated, and it is found that in the spherical and straight-chain molecular films they exhibit regular oscillations, while for the branched alkane their variation is irregular and of a more monotonic nature. These results correlate with structural characteristics of the films, with those of the spherical and straight-chain alkanes exhibiting enhanced layered ordering and in-plane ordered molecular arrangements, as well as with the relatively high tendency for interlayer molecular interdigitation in the squalane films. The nature of the transitions between well-formed layered configurations is different in these systems, with the spherical and straight-alkane films exhibiting solid-like characteristics portrayed by steplike variations in the number of confined segments occurring in response to a small decrease in the gap width, starting from well-layered states of the film. On the other hand, the behavior of the squalane film is liquid-like, exhibiting a monotonic continuous decrease in the number of confined segments as the gap width is decreased. Reduced conformational (trans-guache) transition rates in the confined films, compared to their bulk values, are found, and their oscillatory dependence on the degree of confinement is analyzed, showing smaller transition rates for the well-formed layered states of the films.
\end{abstract}

\section{Introduction}

Understanding the structure, dynamics, and rheology of ultrathin films (of nanometer scale thickness) of low molecular weight hydrocarbons adsorbed on solid surfaces, and in particular of such films confined between solids, is of fundamental interest as well as practical importance for many processes, such as lubrication, adhesion, coatings, chromatography, and membrane separation. Indeed, the properties of such systems have been the subject of numerous investigations (for recent reviews see refs 1-5) employing sensitive microscopies (the surface force apparatus (SFA), atomic force microscopy (AFM), and friction force microscopy (FFM)), computer simulations, ${ }^{1,6-20}$ and other theoretical approaches. ${ }^{3,21-26}$

One of the central issues in this area pertains to the dependencies of the properties of thin confined complex molecular films (e.g. short to medium length alkanes, and polymers) on the nature of the constituent molecules (e.g. molecular weight, straight vs branched chains) and correlations between their response to external parameters (e.g. degree of confinement, load, shear rate) and molecular characteristics. ${ }^{1,20,21,27,28}$ Such issues, which are of fundamental interest, as well as of significance for formulation of molecular-scalebased technological design principles, motivated our study. Moreover, we show that through such systematic investigation (that is, variations of confined film properties with molecular

\footnotetext{
${ }^{\otimes}$ Abstract published in Advance ACS Abstracts, May 1, 1997.
}

shape and complexity) one gains deep insights into the molecular origins of certain fundamental properties of such systems.

Certain key features of confined liquids pertinent to our current study, extracted from a large body of available literature, may be summarized as follows.

(i) Liquids confined between solid boundaries that are smooth on the molecular scale (as well as those adsorbed on a solid surface) tend to organize into layered structures, ${ }^{1,21}$ where the mean local density of the liquid oscillates with distance normal to the boundaries.

(ii) Oscillatory solvation forces between confining surfaces ${ }^{21}$ (that is, oscillations in the force between the confining boundaries as the distance between them, i.e. the confining gap width, is varied, with a period approximately equal to the molecular width) were observed and calculated for simple liquids ${ }^{6}$ (e.g. modeled as spheres), nonpolar globular molecules $^{28-30}$ (e.g. octamethylcyclotetrasiloxane, OMCTS), straightchain alkanes ${ }^{9-13,18,21,31}$ (e.g. $n$-hexadecane, $n-\mathrm{C}_{16} \mathrm{H}_{34}$ ), and even chain molecules with a single pendant methyl group, ${ }^{28,32-34}$ such as 3-methylundecane, $\mathrm{C}_{12} \mathrm{H}_{26}$. On the other hand solvation force measurements ${ }^{28,35}$ for a longer and more heavily branched molecule (i.e. 2,6,10,15,19,23-hexamethyltetracosane, or squalane, a molecule with a $\mathrm{C}_{24}$ backbone and six, symmetrically placed, methyl side groups) confined between mica surfaces have revealed disappearance of the force oscillations, with a sole broad attractive minimum at a gap width $D=18 \AA$, and what appears as a hard wall repulsion at $D=16 \AA$ (measurements 
for smaller gap widths have not been attempted $\left.{ }^{28}\right)$. Recent MD simulations on this system, ${ }^{20}$ extending to even smaller values of $D$, yielded results in agreement with these measurements. From these observations it has been concluded that branching (via pendant methyl groups) can disrupt oscillatory forces, though the degree of branching must be moderately large.

While layering of confined films and solvation force oscillations are related, the distinction between these two phenomena should be emphasized; ${ }^{21}$ for example, as we discussed previously, ${ }^{20}$ the strong attenuation of force oscillations in squalane films in comparison to those found for films of globular molecules and straight-chain alkanes ( $n$-hexadecane and $n$ tetracosane) is not correlated with reduced layering in the branched molecular films. Rather, the difference in solvation force characteristics between the branched and straight-chain (as well as globular) molecular films originates from different modes of response in these systems to variation of the degree of the confinement, with the former characterized as "liquidlike" and the latter as "solid-like". Furthermore, we elucidate in this study, via calculations of the free energy changes in confined fluids, the combined entropic and potential energy origins of solvation forces ${ }^{21}$ and their oscillations.

(iii) Confined ultrathin films may exhibit two different responses $^{27}$ (in shear, ${ }^{27}$ as well as in response to variation of the normal distance between the confining surface ${ }^{30}$ ): a liquidlike response in which the liquid responds to the deformation by flow, ${ }^{27}$ or spreading (as in drainage measurements ${ }^{36,37}$ ), and a solid-like response characterized by observation of the development of "yield stress" in the confined fluid. ${ }^{38}$ Additionally, recent SFA measurements ${ }^{30}$ on confined films of OMCTS (whose diameter is $\sim 9 \AA$ ) suggest the abrupt development of solid-like behavior in relatively thick films of six layers, induced solely by the increased confinement (i.e. without an imposed lateral motion of the confining surfaces).

(iv) Liquid dynamics at interfaces and particularly under confinement is influenced in a marked way, becoming more "sluggish" in nature with increased confinement of the fluid. ${ }^{27}$ Furthermore, molecular architecture and complexity appear to influence the dynamics of confined liquids. ${ }^{39}$

On the theoretical front interfacial and confined liquids have been investigated using both analytical methods ${ }^{21-26}$ and computer-based simulations. ${ }^{6-20}$ In particular molecular dynamics (MD) and Monte Carlo (MC) simulations allow direct investigation of such complex systems with atomic-scale spatial and temporal (using MD) resolution. In these simulations the confining surfaces were modeled as structureless boundaries or as atomically structured solid surfaces, with the confining surfaces and the confined liquids extended indefinitely, or alternatively the liquid has been modeled as a confined droplet ${ }^{34}$ (or semidroplet ${ }^{10}$ ). Extensive simulations have also been performed for complex liquids adsorbed on atomically structured solid surfaces, interacting with tips ${ }^{9,11,12}$ (modeling AFM and FFM experiments).

The configuration of both SFA and tip-based experiments is that of a confined liquid in thermodynamic equilibrium with a surrounding bulk fluid (or unconfined film). Consequently, the measured force (or pressure) is more appropriately described as a disjoining force (or pressure), which is the difference between the force (pressure) in the confinement and that of the bulk fluid with which the confined film is in equilibrium. ${ }^{21,22}$ Therefore it is desirable to conduct simulations of such systems under grand canonical (GC) ensemble conditions (i.e. constant chemical potential $\mu$, pressure $P$, and temperature $T$ ).

Recently, we have formulated and implemented a grand canonical $(\mu P T)$ molecular dynamics (GCMD) method for

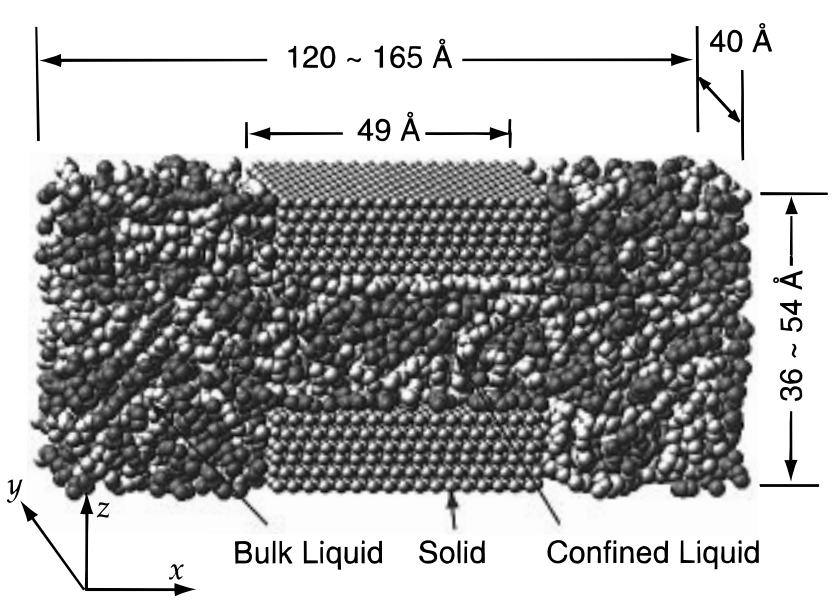

Figure 1. View of the three-dimensional (3D) computational cell for grand canonical molecular dynamics simulations of confined liquids. The cell is repeated using 3D periodic boundary conditions. It contains solid substrates (small spheres) of finite extent in the $x$ direction and extending through the cell in the $y$ direction. The dimension of the cell in the $x$ direction $\left(H_{x}\right)$ varies dynamically in response to the applied external pressure in that direction, taking different values depending on the gap width between the opposing solid surfaces. The cell is filled with liquid molecules; part of them are in the confinement and the rest outside it. $H_{x}$ is taken to be large enough such that bulk liquid behavior can be established in the regions outside the confinement.

simulations of confined lubricants of complex molecular structure. $^{20}$ The method that we developed combines constant pressure MD with a computational cell containing both bulk and confined fluid regions, allowing systematic GCMD investigations of confined liquids and alleviating certain difficulties encountered in grand canonical ensemble MC methods (associated with insertion of molecules), ${ }^{40,41}$ which at the densities of interest to us and for the complex molecules that we use would pose significant practical difficulties.

In this paper, we extend our study to a comparative investigation of structure and solvation force oscillations in a sequence of confined films portraying a trend of increasing complexity: (i) spherical molecules modeled as isotropic Lennard-Jones (LJ) particles, (ii) $n$-hexadecane, representing straight-chain short molecules, (iii) $n$-tetracosane $\left(n-\mathrm{C}_{24} \mathrm{H}_{50}\right)$, representing longer straight-chain molecules, and (iv) squalane (hexamethyltetracosane), which allows a comparative study of branching effects.

In section 2, we describe briefly the formulation and implementation of our GCMD method. ${ }^{20}$ Results of comparative simulations for the four systems listed above are presented in section 3 , including free-energy calculations and a discussion of the energetic and entropic contributions to solvation forces, followed by a summary given in section 4 .

\section{Grand Canonical MD for Confined Liquids}

An overview of the simulated system is shown in Figure 1. The simulation cell contains both liquid molecules and solid blocks, with periodic boundary conditions extending the system in all three directions. The solid blocks are of finite size in the $x$ and $z$ directions, with the distance between the two solid surfaces in the $z$ direction defining the width of the gap $(D)$ confining the fluid (in our simulations the solid surfaces forming the gap in the middle of the cell are modeled as gold (111) planes). In the $y$ direction, the solid blocks are extended through the whole cell. The rest of the space in this three-dimensional cell is filled with fluid molecules.

To facilitate studies of the properties of the confined thin films, which are in equilibrium with a surrounding bulk liquid, the solid blocks as shown in Figure 1 are arranged to create 
two regions of the alkane fluid. Inside the gap, between the two solid surfaces, the alkanes are confined as a thin film with a thickness $D$ ranging between 10 and $30 \AA$. The size of the cell in the $x$ direction is taken to be sufficiently large so that the molecules in the regions outside the confined one can exhibit bulk liquid behavior. The size of the computational cell in the $z$ direction is varied to give the desired film thickness in the gap. In our simulations the extent of the solid blocks in the $x$ direction is $49 \AA$ and the height of each block is $d_{\mathrm{s}}=13 \AA$; the size of the cell in the $z$ direction $H_{z}$ is thus given by $H_{z}=2 d_{s}$ $+D$, and the size of the cell in the y direction is kept constant, $H_{y}=40 \AA$. The size of the cell in the $x$ direction $H_{x}$ is allowed to vary dynamically, using constant pressure $\mathrm{MD},{ }^{42}$ from $\sim 120$ to $\sim 165 \AA$, depending the gap width $D$. The hydrostatic pressure, determining the magnitude of $H_{x}$, is taken to be $1 \mathrm{~atm}$; on the scale of all other stresses in the system this represents a vanishingly low pressure.

In this study, the alkane molecules are treated dynamically, while the gold atoms of the solid substrates are static (treating the solids dynamically does not affect our results); the solid gold blocks contain a total of 3520 atoms, which are immersed in the fluid. The equations of motion of the alkane segments are solved by the Verlet algorithm with an integration time step of $3 \mathrm{fs}$. The temperature of the system is controlled via scaling of the atomic velocities at infrequent intervals (when equilibrium is reached, no temperature control is necessary); the temperature in the simulations involving alkanes is $350 \mathrm{~K}$, which allows the systems to equilibrate in a reasonable amount of computing time, and in the simulations of LJ films $k_{\mathrm{B}} T=0.835 \epsilon$, where $\epsilon$ is the interatomic LJ potential well depth. For the LJ film the 6-12 LJ parameters that we used are $\epsilon / k_{\mathrm{B}}=119.8 \mathrm{~K}$ and $\sigma=$ $3.405 \AA$.

The alkane molecules are represented by the united atom model, without rigid constraints. The intramolecular potentials include bond stretching, bond angle bending, torsional angle, and Lennard Jones (LJ) interactions between all atom pairs separated by more than three bonds. The bond stretching is represented by a stiff linear spring, with the bond force constant reduced by a factor of 4 from its realistic value to facilitate computations with an increased time step (this has no effect on our results). The angle bending, torsional potentials, and LJ potentials describing intra- and interalkane interactions are the same as those used by Mondello and Grest ${ }^{43}$ in their recent study of $n$-alkanes and squalane. ${ }^{44} \mathrm{~A} \mathrm{LJ}$ potential is used to describe the interactions between the alkane segments and the gold atoms of the solid substrates, with parameters fitted to experimental desorption data, as described by us previously. ${ }^{10,45}$

Following an initialization procedure (for details see ref 20), each of the systems has been equillibrated for a long period for a relatively large value of the film thickness $D$, which is the distance between the two solid surfaces confining the films. For subsequent values of $D$, the squalane systems were equilibrated for $300-1000 \mathrm{ps}$, followed by a simulation period of $120-300$ ps, during which the computed properties are time averaged; for the $n$-hexadecane systems and for $n$-tetracosane systems, the equilibration periods are usually $60-480 \mathrm{ps}$ and the data acquisition periods are typically 120 ps (the longer equilibration periods were used for the thinner films). The time histories of several energetic and structural properties were monitored to assure that equilibration has been achieved after each adjustment of the gap width; such adjustments involve squeezing of the film to the next gap width through a slow decrease of $D$, followed by a prolonged equilibration period at the required gap width as described above. During the squeezing process and the first part of the equilibration, the number of molecules

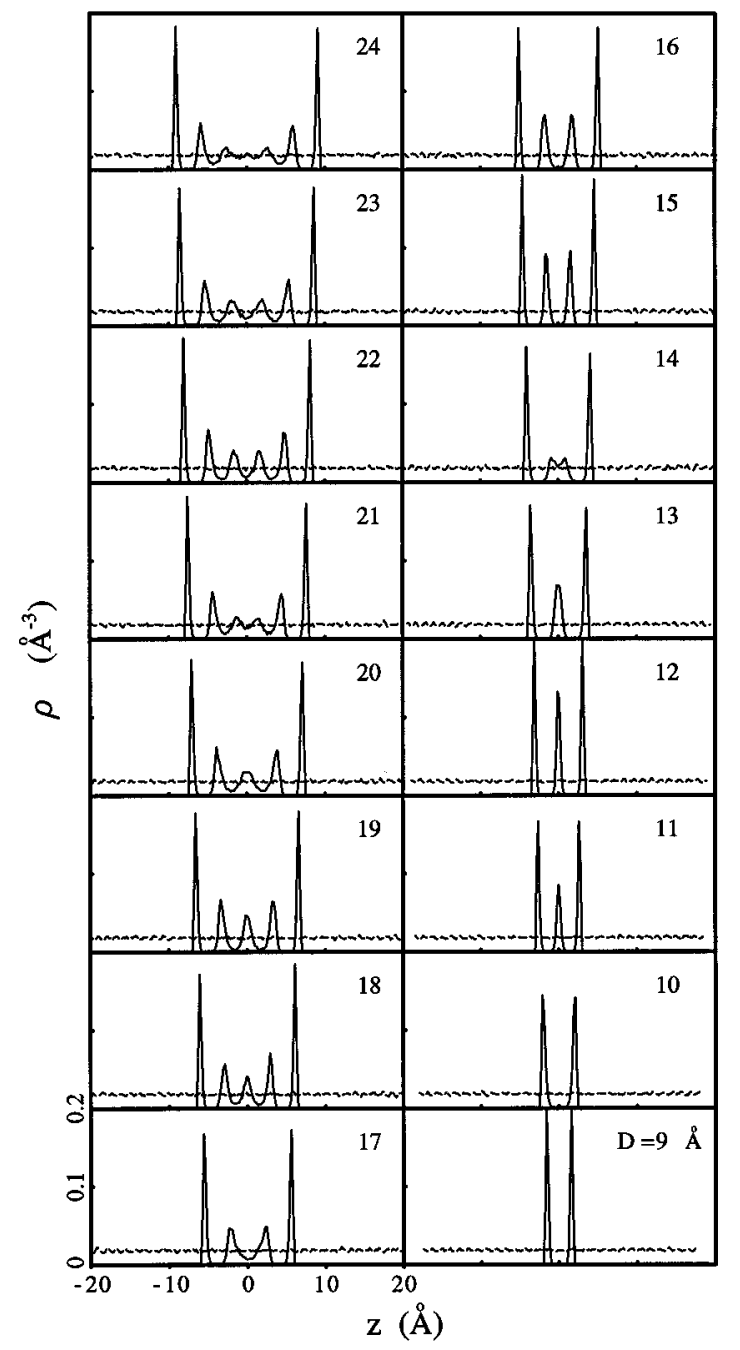

Figure 2. Equilibrium segmental density profiles for LJ fluid along the $z$ direction (normal to the confining solid surfaces). Solid lines correspond to the film in the confined region, and the dashed lines are calculated for the region outside the gap. The gap widths $(D)$ for which the profiles were calculated are indicated. The well-formed layered configurations (see maxima in the solvation force curve in Figure 9) correspond to $D=8,12,15,18,22 \AA$. The other equilibrium profiles were calculated for intermediate values of $D$. Note that the liquid density outside the confinement (dashed line) is uniform and remains constant for all values of $D$, while the layered density features in the confined film (particularly in the middle region of the film) vary in sharpness depending on the degree of confinement, becoming the sharpest for the values of $D$ corresponding to well-formed layers. Distances in units of angstroms.

in the confined region decreases as some molecules are pushed into the bulk region where the pressure remains constant via dynamical variation of $H_{x}$. For the squalane film, reversal of the process at several values of the gap width (i.e. decreasing the confinement) resulted in only slight hysteresis in calculated properties (e.g. solvation force), confirming adequate equilibration.

\section{Results}

Using the new simulation method described in section 2, we have investigated the structures and properties of interfacial LJ and alkane films confined between two opposing surfaces modeled to represent $\mathrm{Au}(111)$ surfaces. As described in section 2 , the confined films are in equilibrium with the surrounding liquid in which the confining solids are immersed.

(a) Layering Transitions and Structures. We begin by presenting our results for the segmental density profiles $\rho(z)$ 


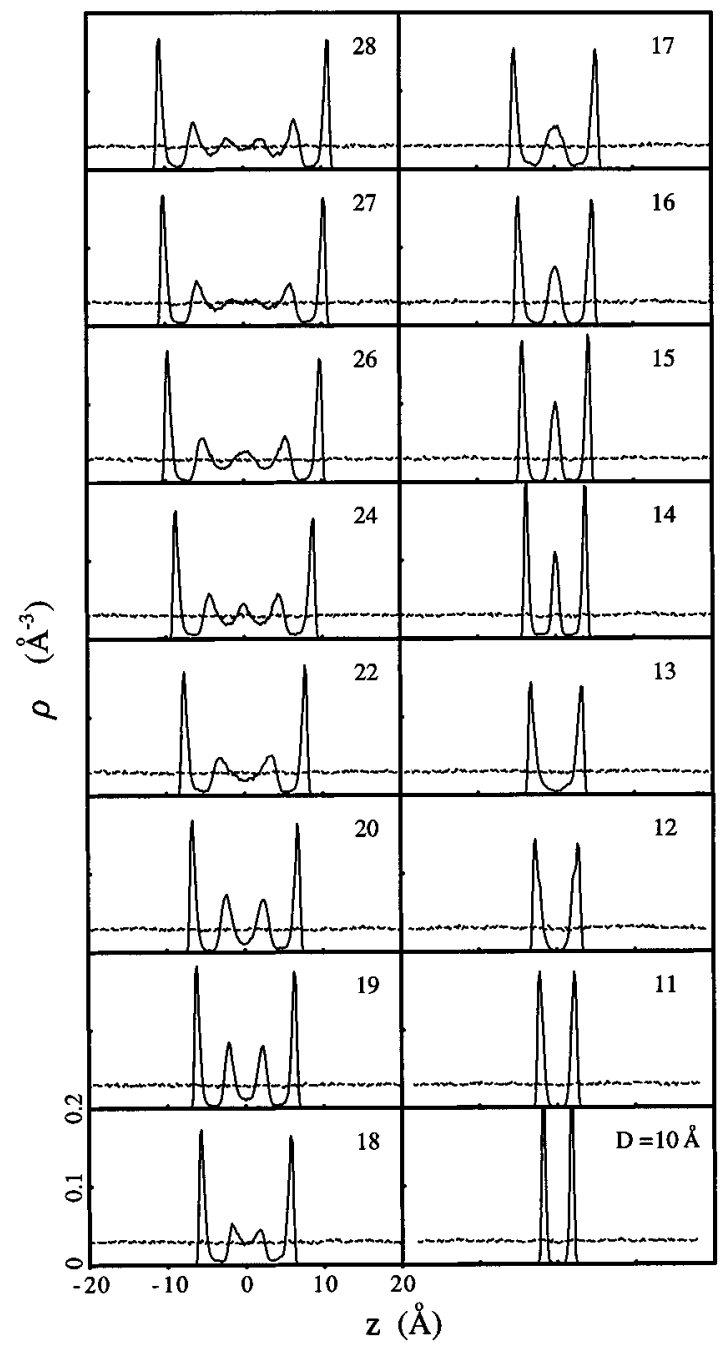

Figure 3. Same as Figure 2, but for $n$-hexadecane. The well-formed layered configurations (see maxima in the solvation force curve in Figure 9) correspond to $D=10,14,19,24 \AA$.

for the two systems, recorded vs distance in the direction normal to the surfaces, for a sequence of separations (gaps) between the confining surfaces (see Figures 2-5).

While the density profiles (Figures 2-5) for all materials exhibit oscillatory patterns, a closer inspection reveals some differences. Already for large separations between the surfaces (a wide gap, $D=22 \AA$ for the LJ film and $D=28 \AA$ for the alkanes) the LJ and straight-chain alkane films appear to be well layered throughout with six well-formed layers (Figure $2-4$, top left), while for the same gap width the squalane film exhibits only four well-formed layers and in the middle region of the gap the density is close to that in the bulk (Figure 5, top left). We also note for the squalane case the appearance of distinct features between the first interfacial layer on each side of the film and the second layer in, which are absent in the straight-chain alkane profile. These features, which may also be seen (though less pronounced) between the second and third layer of the squalane film are caused by the methyl branches that lie preferentially between the layers (a similar behavior has been observed in previous simulations of a single-branched hexadecane film ${ }^{34}$ ).

Decreasing the width of the gap is accompanied by reduction of the number of layers in the film (see Figure 2 for $D=18 \AA$ and Figures 3-5 for $D=24 \AA$, where five well-formed layers are observed for all films). We also remark that the density minima between the layers are deeper for the LJ and straightchain alkanes than in squalane.

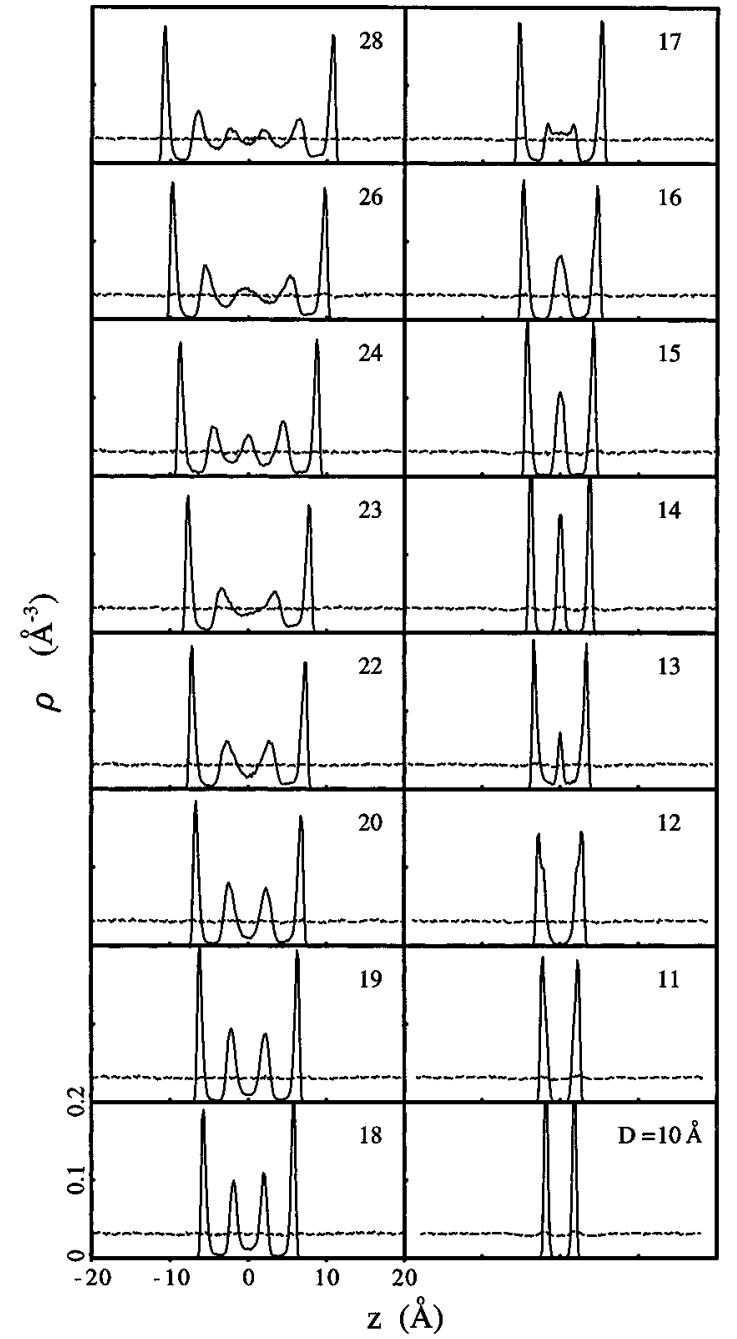

Figure 4. Same as Figure 2, but for $n$-tetracosane. The well-formed layered configurations (see maxima in the solvation force curve in Figure 9) correspond to $D=10,14,18,24 \AA$.

While organization of the confined film density into layered structures, with the number of layers dependent on the gap width, occurs in all the systems studied here, the degree of order in the alkane films varies, with that in the straight-chain ones being higher. This may be ascertained from close inspection of the density profiles shown in Figures 3-5, where the minima between layers are deeper for $n$-hexadecane and $n$-tetracosane, as well as from visual inspection of molecular configurations of the films (see molecular configurations in ref 20), exhibiting enhanced layering order in the straight-chain alkane films as compared to the squalane one. The degree of in-plane intermolecular order in the first layer (interfacial layer closest to the solid surface) in these films is clearly larger in the linear alkane films (similarly for the second layer) where ordered domains are found (see Figure 7 in ref 20, showing results for $n$-hexadecane; similar results were found by us for $n$-tetracosane).

A high degree of intralayer order is found also for the spherical (i.e. LJ) molecular confined films (for earlier investigations of in-layer order in confined LJ films, see refs 4649). In particular, in these films, in-layer ordering extends further into the confined film (that is, beyond the first and second interfacial, boundary, layers). Average pair distribution functions for equilibrated LJ films with $D=15,14$, and $12 \AA$ (with those for 15 and $12 \AA$ corresponding to well-formed layered configurations containing four and three layers, respectively) are shown in Figure 6 (the corresponding density profiles are 


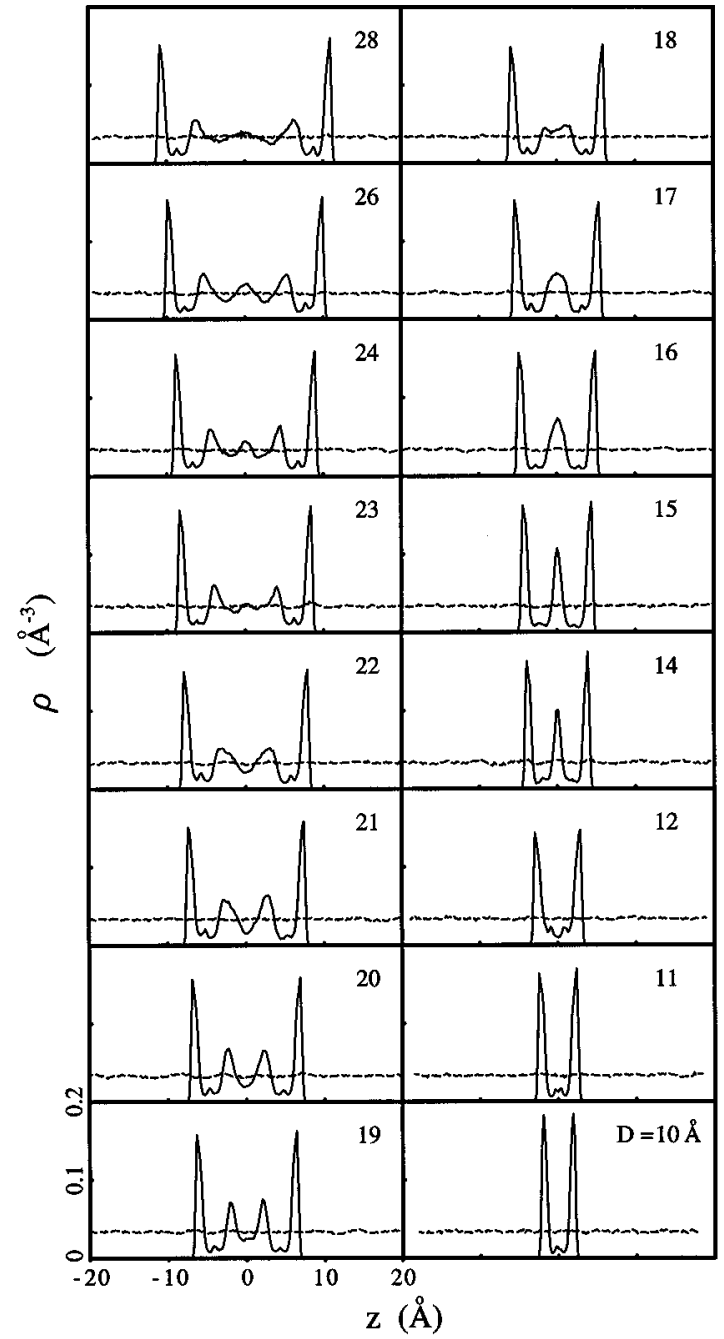

Figure 5. Same as Figure 2, but for squalane. The well-formed layer configurations correspond to the maxima in the solvation force shown in Figure 9. Note that small local density maxima on each side of the confined film, located between the interfacial layer and the next layer in; such a local maximum between the interfacial layers is seen even for the two-layer film configurations. The local density minima between layers are not as deep as those found for the spherical (LJ) and straightalkane films (compare to corresponding configurations in Figures 3, 4). Distances in units of angstroms.

given in Figure 2). We observe that for the four-layer configuration (Figure $6 \mathrm{a}$ ) both the interfacial and middle layers exhibit a high degree of order (inspection of molecular images reveals hexagonally close-packed domains with defects). The degree of order in the middle region of the film is significantly reduced upon reducing the gap width by merely $1 \AA$ (see Figure $6 \mathrm{~b})$. However upon further decrease of the gap-width to $D=$ $12 \AA$, corresponding to a well-formed three-layer film, in-layer order is restored, with the structure of both the interfacial and middle layer exhibiting an almost perfect hexagonal-closepacked arrangement.

Statistical analysis of the results obtained for the alkane films shows (see Table 1) that for the four-layer films the probabilities, $f_{\mathrm{ee}}^{(1, i)}$, for one end of a molecule to be in the interfacial layer (designated as the first layer; note that by symmetry the interfacial layers on both sides of the film are equivalent, therefore we average our results for both interfaces) and the other end to be located either at the first, second or third layer ( $i=1,2,3$ respectively) are almost evenly distributed for squalane, indicating a high degree of interlayer interdigitation (bridging) in the longer branched alkane film. On the other hand, for the straight-chain alkanes the probability distribution

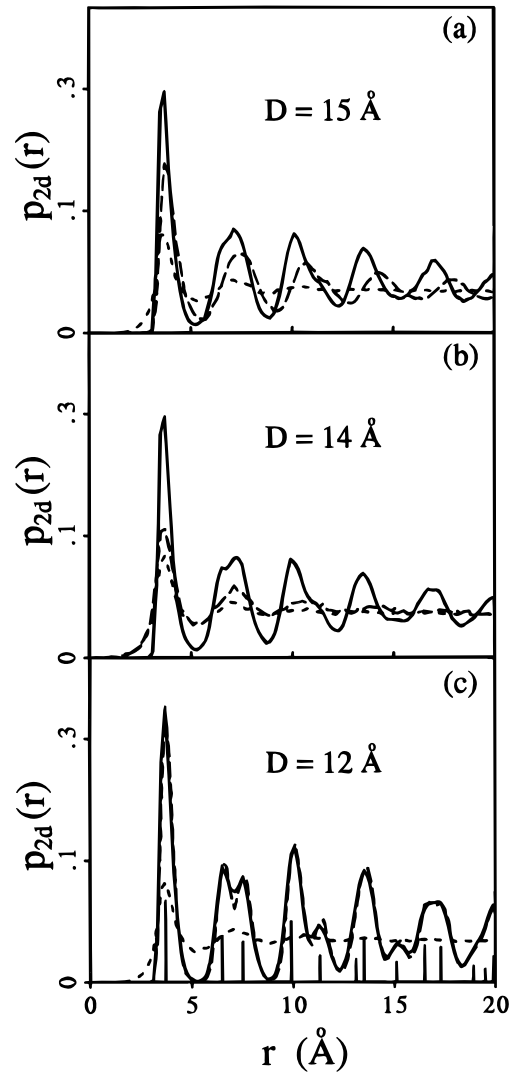

Figure 6. Equilibrium averaged two-dimensional pair distribution functions, $p_{2 d}(r)$, calculated for three configurations of LJ confined films, with gap width $D=15 \AA$ (a), $14 \AA$ (b), and $12 \AA$ (c). The configurations in a and c correspond to well-formed layered films with four and three layers, respectively (see Figure 2). Solid lines represent results for the interfacial layer interacting directly with the solid surface, long-dashed line corresponds to the middle layers in the confined film, and shortdashed lines correspond to $p_{2 d}(r)$ calculated in the bulk region (i.e. outside the confinement). Note the obliteration of order in the middle region of the confined film, in the intermediate equilibrium film with $D=14 \AA$ (b). In c the $p_{2 d}(r)$ curves for the interfacial and middle layers essentially coincide. The vertical lines at the bottom denote the positions (and weights) corresponding to a perfect $2 \mathrm{D}$ hexagonally close-packed structure of LJ particles of similar density as that in the layers of the confined film shown in c.

is dominated by both ends of the molecules being located in the interfacial layer. Additionally, for molecules with one end in one of the middle layers (designated as layers 2 and 3) the probabilities, $f_{\mathrm{ee}}^{(2, i)}$, that one end of the molecule is in one of these layers and the other is found either in the same layer or in the other one show a higher degree of interlayer interdigitation for molecules in the middle layers of the films, with a larger degree for the squalane film. A similar analysis but for the probabilities, $f_{\mathrm{em}}^{(1, i),}$ of one end of a molecule to be in the interfacial layer (layer 1 or 4 ) and the middle segment of the molecule to be located either in the same layer or in the middle layers of the film (layers 2 and 3) indicate that for the straight alkane films the small amount of interlayer interdigitation involves mainly the molecular tails, while for the squalane film a larger part of the molecule may be distributed between neighboring layers; the corresponding probabilities for the middle layers of the films are denoted by $f_{\mathrm{em}}^{(2, i)}$ in Table 1 . Analysis of the three-layer films (see Table 1) shows similar trends; in particular, for the squalane film the end-end probabilities show that over $10 \%$ of the molecules bridge the two interfaces in the three-layer squalane system.

The degree of order in the alkane films can also be analyzed in terms of the order parameter $P_{2}(\cos \theta)=\left\langle\left(3 \cos ^{2} \theta-1\right) / 2\right\rangle$, 
TABLE 1: Equilibrium Averaged Layer Occupation-Conformational Probabilities in Four- and Three-Layer Confined Films of Straight-Chain Alkanes ( $n$-Hexadecane and $n$-Tetracosane) and of Squalane ${ }^{a}$

\begin{tabular}{ccccc}
\hline & $i$ & $n$ - $\mathrm{C}_{16} \mathrm{H}_{34}$ & $n-\mathrm{C}_{24} \mathrm{H}_{50}$ & squalane \\
\hline \multirow{3}{*}{$f_{\mathrm{ee}}^{(1, i)}$} & 1 & 4 Layers & & \\
& 2 & 0.80 & 0.74 & 0.33 \\
& 3 & 0.12 & 0.17 & 0.37 \\
$f_{\mathrm{ee}}^{(2, i)}$ & 4 & 0.00 & 0.06 & 0.30 \\
& 2 & 0.60 & 0.02 & 0.00 \\
$f_{\mathrm{em}}^{(1, i)}$ & 3 & 0.40 & 0.73 & 0.38 \\
& 1 & 0.90 & 0.27 & 0.62 \\
& 2 & 0.08 & 0.08 & 0.50 \\
$f_{\mathrm{em}}^{(2, i)}$ & 3 & 0.02 & 0.02 & 0.39 \\
& 2 & 0.00 & 0.00 & 0.11 \\
& 3 & 0.79 & 0.86 & 0.00 \\
$f_{\mathrm{ee}}^{(1, i)}$ & 1 & 0.21 & 0.14 & 0.37 \\
& 2 & 0.90 & 0.98 & \\
$f_{\mathrm{em}}^{(1, i)}$ & 3 & 0.10 & 0.02 & 0.32 \\
& 1 & 0.94 & 0.00 & 0.55 \\
& 2 & 0.06 & 0.97 & 0.65 \\
& 3 & 0.00 & 0.03 & 0.30 \\
& & & 0.00 & 0.05
\end{tabular}

${ }^{a}$ The layers denoted $i=1$ and 4 (for the four-layer films) and $i=$ 1 and 3 (for the three-layer films) correspond to the interfacial layers in direct contact with the confining solid surfaces. Layers 2 and 3 (for the four-layer films) and layer 2 (for the three-layer films) are the middle layers in the confined films. $f_{e e}^{(n, i)}$ is the probability that one end of a molecule is found in layer $n$ and the other end in layer $i$. $f_{\mathrm{em}}^{(n, i)}$ is the probability that one end of a molecule is found in layer $n$ and the middle of the molecule in layer $i$. Note the high degree of interlayer interdigitation for the squalane films.

where $\theta$ is the angle formed between bond vectors connecting neighboring segments of the molecules and the direction normal to the surface, and the angular brackets denote averaging over all bonds in the regions (confined or bulk) and over the MD simulation. The results displayed in Figure 7 show that in the bulk regions the molecular bonds do not exhibit any orientational order, i.e. $P_{2}(\cos \theta) \approx 0$. On the other hand in the confined films they show a tendency to lie preferentially parallel to the confining surfaces with the degree of that preferred orientation being larger in the straight-chain alkanes films. Furthermore, the degree of preferential orientational order (i.e. more negative values of $\left.P_{2}(\cos \theta)\right)$ oscillates as a function of the gap width, with local maximal orientational ordering achieved for gap widths corresponding to well-formed layers (i.e. solvation force maxima, see below), with intervening states of reduced orientational order corresponding to the transitions between layered states of the films as $D$ is varied.

Finally, we show in Figure 8 the torsional angle transition rates between trans and gauche conformations of the alkane molecules. We observe that in the bulk regions of $n$-hexadecane and $n$-tetracosane the transition rates are higher than in the bulk squalane liquid. The transition rates in the confined systems are lower than their corresponding bulk values and exhibit an oscillatory behavior as a function of the gap width, with local minima of the transition rates occurring for values of $D$ corresponding to well-formed layers. The amplitudes of the oscillations of the transition rates vs $D$ are larger for the $n$-hexadecane and $n$-tetracosane films, and they increase as the confining gap width is reduced.

These results correlate with those discussed above pertaining to structural properties, showing that both structural and dynamical characteristics of complex liquids are influenced greatly by their confinement. Moreover, our simulations

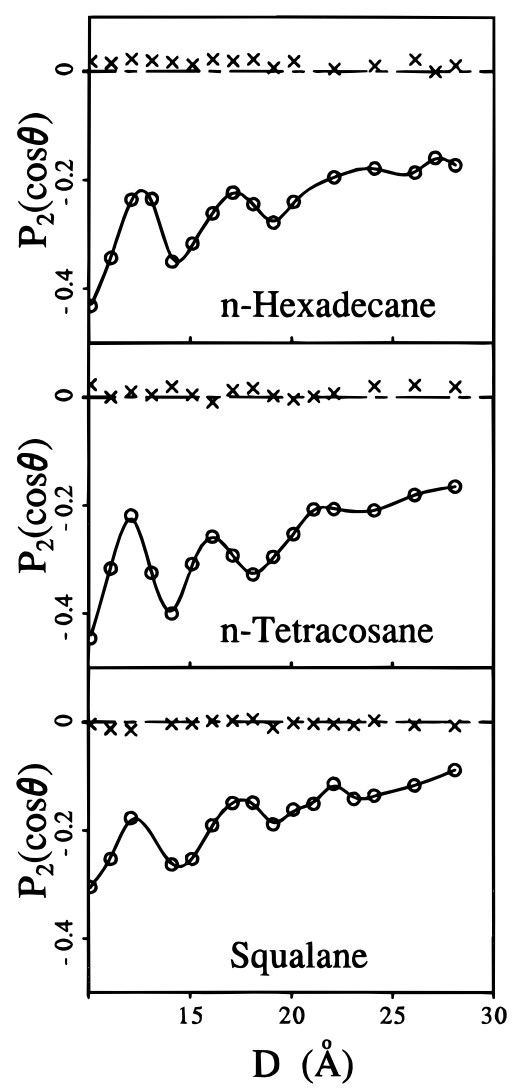

Figure 7. Segment bond order parameter, $P_{2}(\cos \theta)$, plotted vs the gap width, $D$ in angstroms, for the equilibrium states of the $n$-hexadecane, $n$-tetracosane, and squalane systems. The crosses correspond to values obtained for the bulk liquids outside the confinement, and the solid lines connect values calculated for the confined films.

revealed dependencies of the various degrees of order in the films (layering, in-plane order, and orientational ordering) on the complexity of the molecular constituents and on the degree of confinement.

(b) Solvation Forces. Narrowing of the gap results in expulsion of molecules from the confined region ("squeezingout" of the film) and transition to a film with a smaller number of layers (which remain throughout to be of similar density, i.e. containing approximately the same number of molecular segments), occurring with a periodicity of about $4.5-5 \AA$. Solvation forces $f_{z}(D)$ (i.e. the total force exerted by the interfacial film on the confining surfaces, which is the same as the force that would be required in order to hold the two surfaces at the corresponding separation) recorded for the four systems during the approach of the the two surfaces are shown in Figure 9. Additionally we display in Figure 10 records of the number of molecular segments (Figure 10a) confined in the gap between the two surfaces, $n_{\mathrm{cfn}}$, as a function of the width of the gap, $D$, as well as of $n_{\mathrm{cfn}} / D$ plotted vs $D$ (Figure 10b).

The layering transitions in the confined films are portrayed in solvation force oscillations (Figure 9). However, while for the LJ and straight-chain alkanes the force oscillates between positive and negative values, with the local positive force maxima corresponding to configurations with well-formed layers, in the squalane case at wider gaps the force does not take negative values, and the amplitudes of the oscillations are smaller than in the hexadecane case. For narrow gaps $(D \lesssim 15 \AA)$ the force maximum associated with a transition from a three-layer to a two-layer film is significantly larger in the squalane film than that in the $n$-hexadecane and $n$-tetracosane ones.

Further insights into the layering transition processes are obtained from records of the number of segments in the confined 


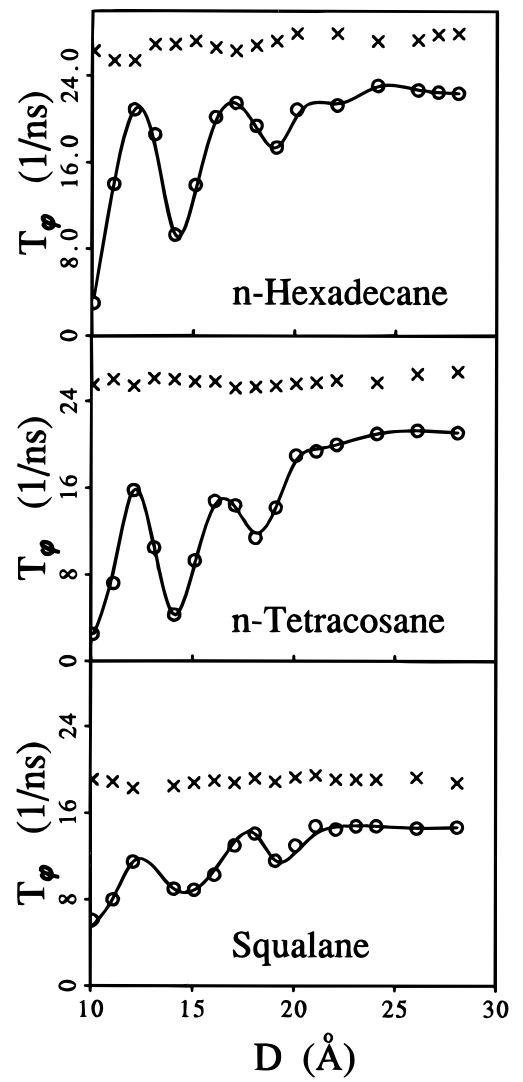

Figure 8. Trans-gauche (and vice versa) transition rates, $T_{\phi}$ (in units of $1 / \mathrm{ns}$ ) plotted vs the gap width, $D$ in angstroms, for the equilibrium states of the $n$-hexadecane, $n$-tetracosane, and squalane systems. The crosses correspond to values obtained for the bulk liquids outside the confinement, and the solid lines connect values calculated for the confined films.

region, $n_{\mathrm{cfn}}$, plotted vs the distance between the confining surfaces $(D)$, shown in Figure 10a. In the LJ film, for $D \leq 22$ $\AA$ (i.e. six-layer film) and in the $n$-hexadecane and $n$-tetracosane films, for $D \leq 20 \AA$, (i.e. four-layer films) $n_{\text {cfn }}$ varies in a steplike manner, with sharp drops in the number of confined molecules occurring for the transition from an $n$-layer film to an $(n-1)$-layer one, with the steps becoming sharper as $n$ decreases. For each of these transitions the drop in $n_{\mathrm{cfn}}$ is caused by a relatively small reduction $(\sim 1 \AA)$ of the gap width from that corresponding to an initial well-layered configuration of the film (that is, the number of layers in the film at the bottom of each step is decreased by 1 from that corresponding to the film at the top of the step (and the plateau). On the other hand, for the squalane film the variation of $n_{\mathrm{cfn}}$ associated with the layering transitions is overall monotonic and continuous in nature, with the development of a weak steplike feature only at the limit of a very thin film (i.e. see transition from a three- to a two-layer film, for $D \lesssim 15 \AA$, in Figure 7a).

These characteristics of the layering transitions are further found (see Figure 10b) in plots of $n_{\mathrm{cfn}} / D$ vs $D$ (where $n_{\mathrm{cfn}} / D$ is proportional to the number density of molecular segments in the gap, since the area of the confining solid surfaces is constant for all values of $D$ ). These plots illustrate that for the squalane film the confined segmental density remains approximately constant throughout the narrowing (squeezing) process (except in the limit of a very thin film, $D \lesssim 15 \AA$ ), while for the other films reducing the gap width is accompanied by marked variations in the density (becoming increasingly more pronounced as the film thins down). Furthermore, in the latter case the local maxima of the film density, corresponding to wellformed layered configurations, are of equal value.
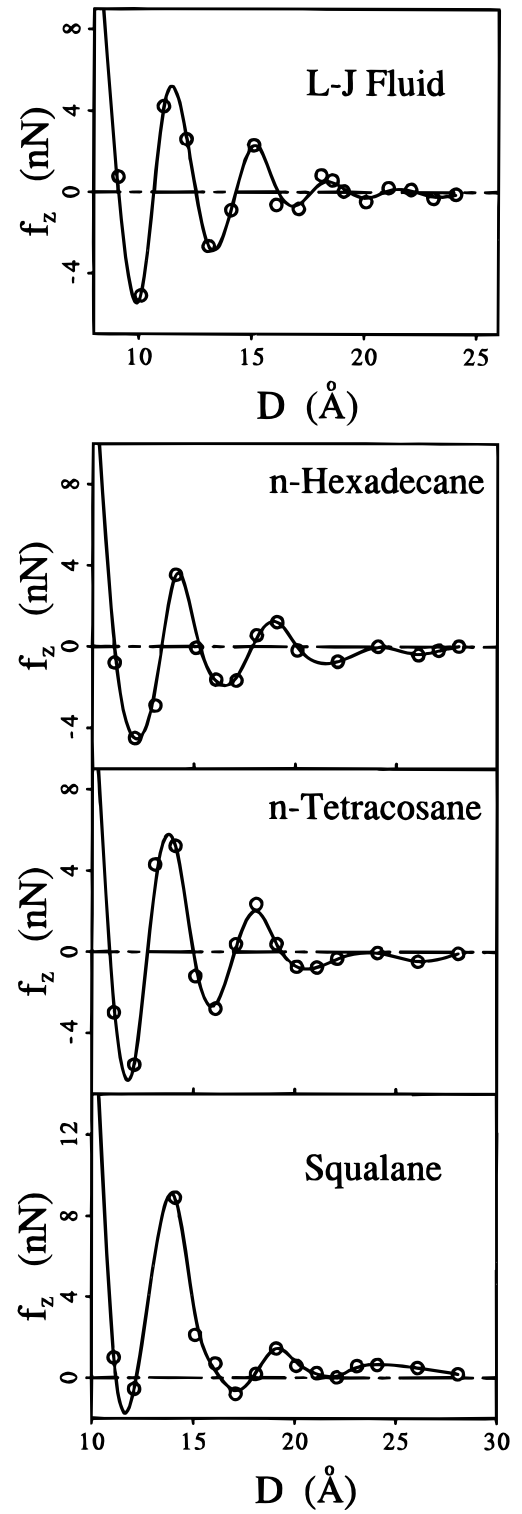

Figure 9. Equilibrium solvation forces, $f_{z}$ in $\mathrm{nN}$, plotted vs the width of the confining gap, $D$ in angstroms, calculated for the confined LJ, $n$-hexadecane, $n$-tetracosane, and squalane films. Note that for the LJ $(D>17 \AA)$, the $n$-hexadecane, and the $n$-tetracosane $(D>20 \AA) F_{z}$ is negative (attractive), while for the squalane film the force is overall repulsive with small local attractive minima near $D$ equal to $\sim 17$ and $\sim 12 \AA$. Well-formed layers occur for $D$ values corresponding to local maxima (positive $F_{z}$ values). The repulsive forces in the squalane film are stronger than in the straight-chain alkane and spherical (LJ) films.

These results suggest that the confined squalane film behaves in a liquid-like manner throughout most of the gap-narrowing sequence; that is, the equilibrium confined film, which is in contact with the surrounding bulk liquid, maintains a constant density for varying widths of the confining gap by gradually expelling molecules into the surrounding liquid when the gap width is reduced. In contrast, confined spherical (LJ) and straight-chain alkane films of sufficiently small thickness exhibit certain features characteristic of solid-like response; that is, when the confining gap width is slightly reduced (typically $\Delta D \approx$ $1-2 \AA$ ), starting from one of the well-formed layered configurations of the film with $n_{\mathrm{L}}$ layers (corresponding to the maxima in the solvation force shown in Figure 9), the film "yields" through expulsion of approximately a layer worth of molecular segments into the surrounding liquid, causing a sharp decrease in the confined film density. During further reduction of the gap width, the number of confined molecular segments remains 

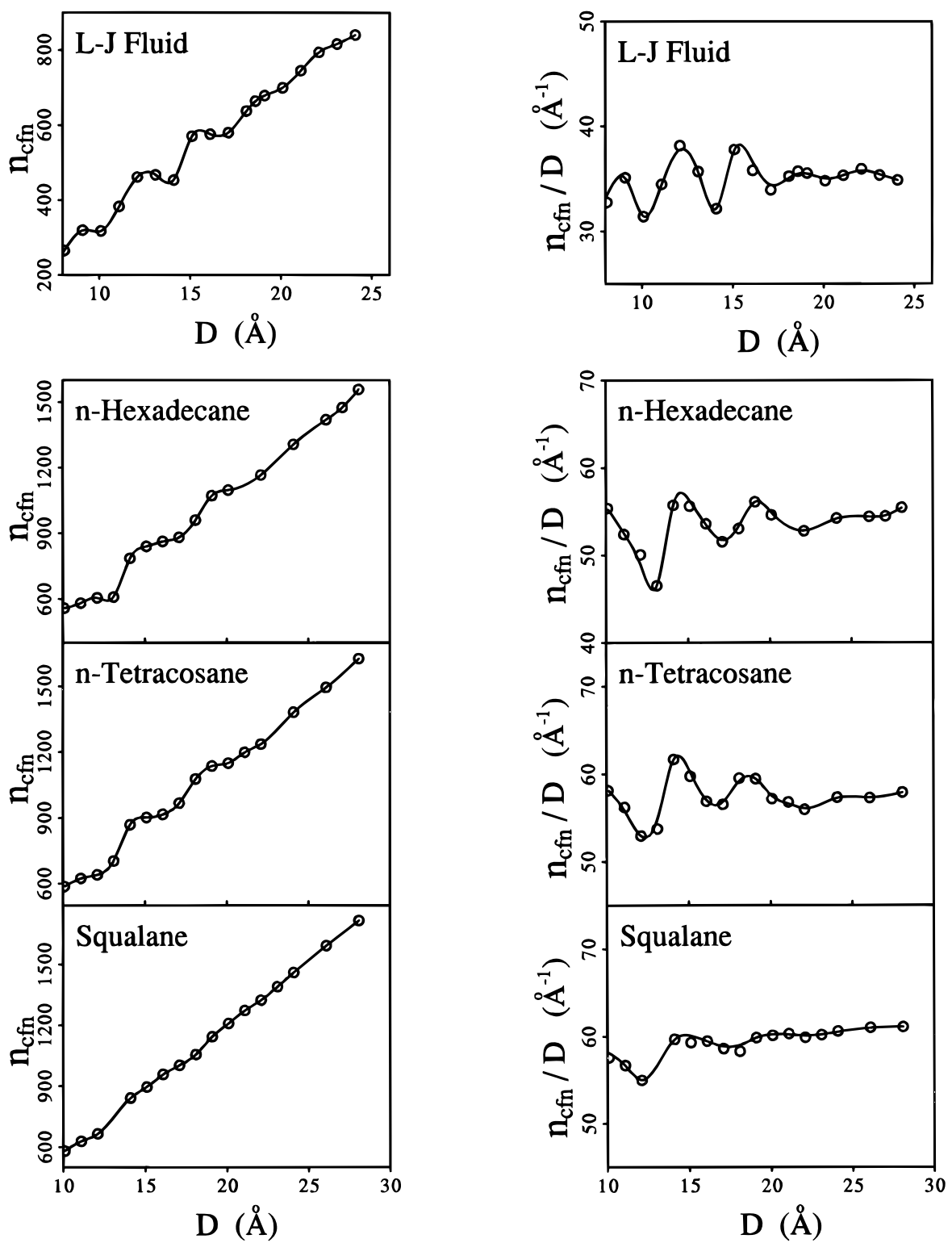

Figure 10. Number of atoms (for LJ) or molecular segments (for alkanes) in the confining gap, $n_{\mathrm{cfn}}$, in part a (left) and $n_{\mathrm{cfn}} / D$, in part $\mathrm{b}$ (right), plotted vs the gap width, $D$ in angstroms, for the four confined film systems.

almost constant (corresponding to the plateaus of $n_{\text {ncf }}$ shown in Figure 10a), with an associated increase of the confined film density (see $n_{\mathrm{cfn}} / D$ in Figure 10b), which is accompanied by enhancement of the order in the layered structure of the film. This process continues until a gap width corresponding to a maximally ordered layered film (with $n_{\mathrm{L}}-1$ layers) is reached, for which the confined film density maximizes. This sequence of events repeats with a period of $\sim 4.5-5 \AA$.

At this juncture we reemphasize that the states of the confined films that we analyze here are all at equilibrium conditions. Consequently, we argue that the different nature of the layering transitions between the LJ and straight-chain alkane films and that found for the squalane film reflects differences in the equilibrium properties of the confined films. In particular, we associate the aforementioned solid-like characteristics with a high degree of order in the the layered LJ and straight-chain alkane confined films, while the liquid-like nature found for the squalane case is correlated with frustration of the order in the confined film, caused by branching.

(c) Entropic and Energetic Contributions to Solvation Forces. Solvation forces originate from changes in the free energy of the systems as the separation between the two confining surfaces is varied. Such changes in the free energy may involve energetic and entropic contributions. ${ }^{21}$ In the following we explore these variations using the results of our GCMD simulations.

It is convenient to analyze our systems when for a particular molecular liquid the number of molecules in the confinement remains constant throughout the approach of the confining surfaces. This can be achieved either through varying the area of the confining surfaces upon changing the gap distance $(D)$ or by scaling extensive properties of the confined fluid simulated at constant area of the confining surfaces. The latter procedure is computationally simpler; accordingly, starting from a system with a wide gap, $D_{0}$, for which the equilibrium number of molecular segments confined in the gap region is $N_{0}$, the total internal energy, $U(D)$, and total force, $f_{z}(D)$, exerted by the confined fluid on the confining surfaces simulated for a gap size $D$ are scaled by multiplying $U(D)$ and $f_{z}(D)$ by $N_{0} / N(D)$, where $N(D)$ is the equilibrium number of molecular segments in the confinement.

The change in the free energy of the confined liquid associated with a change of the gap size from $D_{0}$ to $D$, performed quasi-statically under isothermal conditions and for 

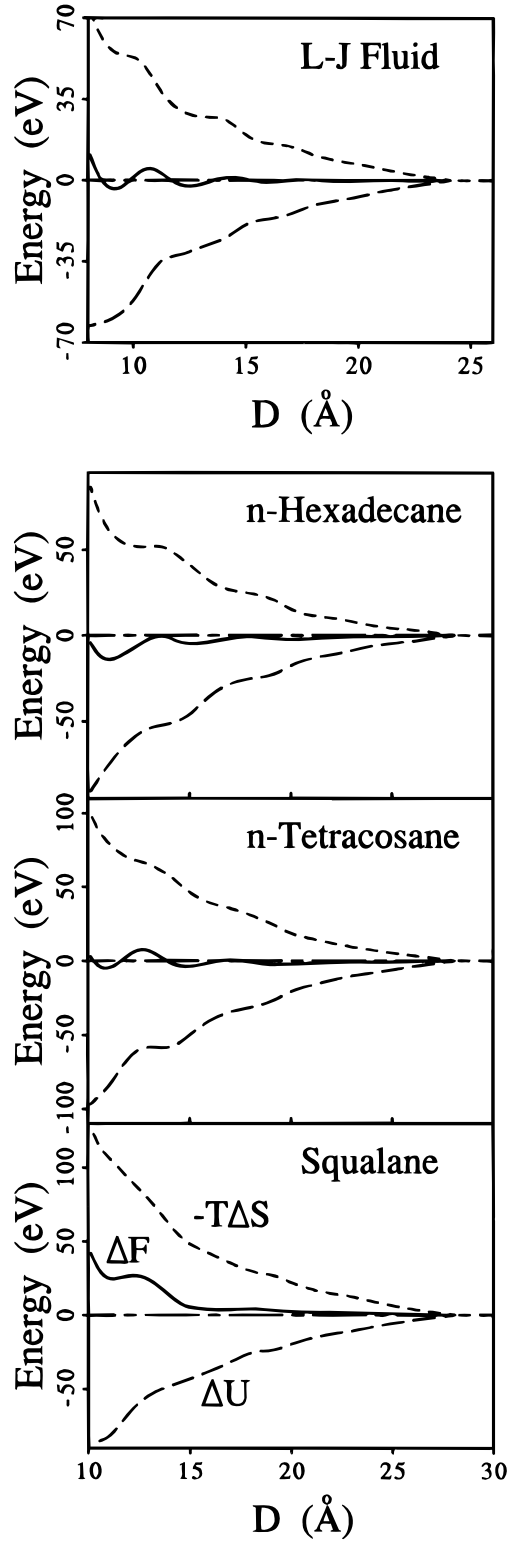

Figure 11. Changes in the free energy ( $\Delta F$, solid line), internal energy

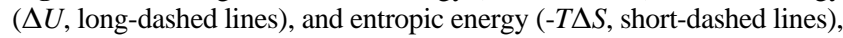
calculated as described in the text, plotted vs the gap width, $D$ in angstroms, for the four confined film systems. Note the overall repulsive behavior of $\Delta F$ for the branched alkane (squalane).

a constant number of confined molecules, is equal to the reversible work done in this process, i.e.

$$
\Delta F\left(D, D_{0}\right)=\int_{D_{0}}^{D} f_{z}(x) \mathrm{d} x
$$

From the definition of $\Delta F$, i.e.

$$
\Delta F\left(D, D_{0}\right)=\Delta U-T \Delta S
$$

where $\Delta U$ and $\Delta S$ are the corresponding changes in the internal energy and entropy of the confined system, one can evaluate the entropic contribution $T \Delta S$ using $\Delta F\left(D, D_{0}\right)$ from eq 1 and the internal energy changes obtained from the MD simulation (scaled as described above). In eq 2 the pressure-volume, $P \Delta V$, contribution has been omitted since both $P$ and $\Delta V$ are small. Results for the internal energies, $T \Delta S$, and free energies, calculated for the systems simulated in this study, are shown in Figure 11. The main features noted from these results are the oscillations in the free energy (which are phase shifted when
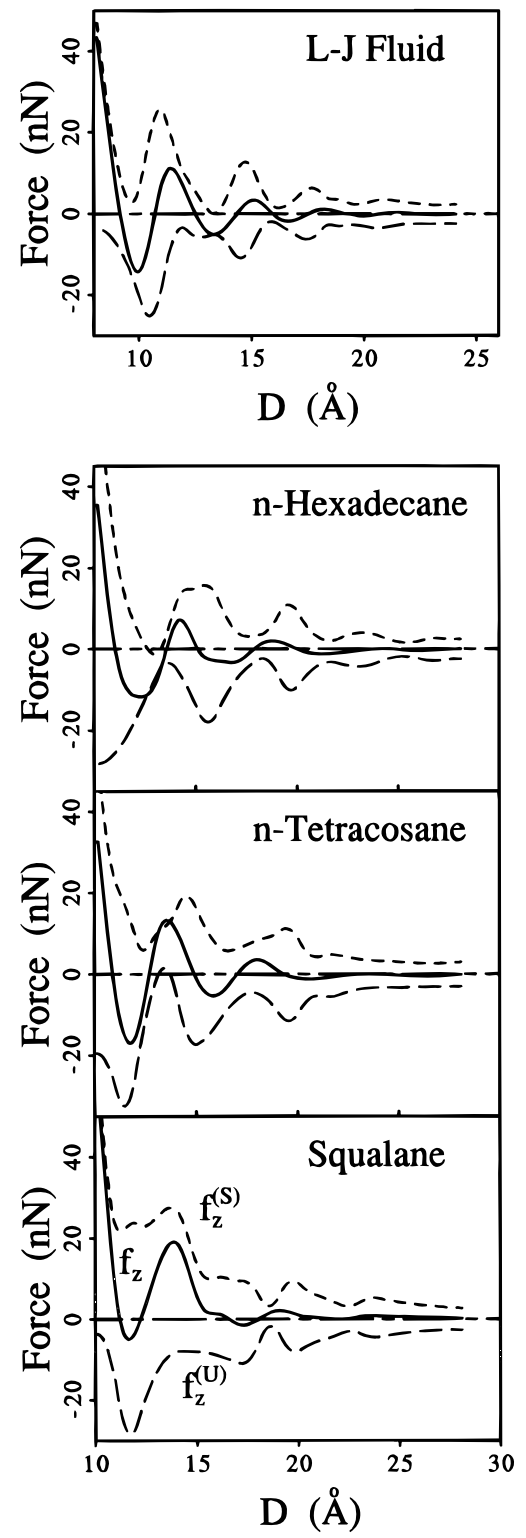

Figure 12. Total solvation force $\left(f_{z}\right.$, solid line), its internal energy component $\left(f_{z}^{(U)}\right.$, long-dashed lines), and the entropic force component $\left(f_{z}^{S)}\right.$, short-dashed lines), calculated as described in the text, plotted vs the gap width, $D$ in angstroms, for the four confined film systems. Note the regular oscillatory behavior of $f_{z}^{(U)}$ and $f_{z}^{(S)}$ for the LJ and the straight-chain alkane films and the more irregular variations of these quantities for the squalane films.

compared to the solvation force oscillations; that is, the extremal values of $\Delta F(D)$ correspond to zeros of $\left.f_{z}(D)\right)$ and the monotonic variations of the potential energy and entropic contribution, with those for the LJ and straight-chain alkane films exhibiting steplike features. We note here that the development of wellformed layered configurations starts at gap widths corresponding to the minima of $\Delta F$, sharpening further as the solvation forces achieve their maximal values.

The internal energy and entropic contributions to the total solvation force $f_{z}(D)$ can be now evaluated as $f_{z}^{(U)}(D)=$ $-\partial(\Delta U) / \partial D$ and $f_{z}^{(S)}(D)=\partial(T \Delta S) / \partial D$, which when combined yield the total solvation force $f_{z}(D)=-\partial(\Delta F) / \partial D$ (see eq 1$)$. These results for the systems under study are shown in Figure 12. We note that in all cases studied here both the internal and entropic contributions to the solvation force exhibit oscillations as a function of the width of the confining gap. The entropic contribution to the solvation force is overall repulsive (positive values), showing local maxima in the vicinity of the well-layered 
configurations, while the internal energy contribution is overall attractive (negative values).

The pattern of variations vs the gap width of the entropic and energetic contributions to the total solvation force in the different films reflects the nature and degree of molecular organization and ordering during the squeezing process. As mentioned previously, the degree of order in the LJ and straightchain alkane films is higher, particularly in the well-formed layered configurations, than in the branched (squalane) film, where both inter- and intralayer ordering is frustrated even in the well-layered stages. Consequently for the former ones both $f_{z}^{(S)}(D)$ and $f_{z}^{(U)}(D)$ exhibit well-defined oscillations corresponding to transitions between well-formed layered configurations, while the variations of these quantities for the squalane film are more monotonic in nature.

\section{Summary}

In this paper a comparative study has been presented of equilibrium structures, solvation forces, and conformational dynamics of thin confined films of spherical molecules (modeled as LJ spheres), straight-chain alkanes of variable lengths ( $n$ hexadecane and $n$-tetracosane), and a branched alkane (squalane), using a recently developed ${ }^{20}$ grand canonical ensemble molecular dynamics method for simulations of confined liquids. The method combines constant pressure simulations with a computational cell containing solid surfaces and both bulk and confined liquid regions in equilibrium with each other (Figure 1), allowing investigations of confined films under conditions similar to those used in surface force apparatus and tip-based experiments.

Our studies provide insights into the nature of equilibrium confined liquids and the effects of molecular structure (shape, chain length, and branching) on the properties of such systems. For all the liquids that we have investigated layered density oscillations in the confined films were found, with the number of layers depending on the width of the confinement. The LJ and straight-chain alkane confined films exhibit enhanced layering order, as well as a higher degree of in-plane molecular ordering, compared to those found for the squalane film, with the latter showing a high tendency for interlayer molecular interdigitation (see Table 1). Reduced conformational (transguache) transition rates in the confined alkane films, compared to their bulk values, are found, and their oscillatory dependence on the degree of confinement is analyzed, showing smaller transition rates for the well-formed layered states of the films (Figure 8).

The solvation force oscillations as a function of the gap width for the spherical and straight-chain alkane liquids are more pronounced, exhibiting attractive and repulsive regions, while for the branched alkane the solvation forces are mostly repulsive, with the development of shallow local attractive regions for small values of the gap width (Figure 9). These results correlate with recent observations using SFA measurement on confined films of squalane, ${ }^{28}$ showing the influence of molecular structure (i.e. moderate degree of branching) on the character of the solvation forces. Furthermore, the nature of the transitions, and equilibrium intermediate states, between well-formed layered configurations is different in the two systems, with the spherical and straight-chain alkane films exhibiting solid-like characteristics portrayed by steplike variations in the number of confined segments occurring in response to a small decrease $(\sim 1-2 \AA)$ in the gap width, starting from well-layered states of the film (Figure 10a). On the other hand the behavior of the squalane film is liquid-like, exhibiting a monotonic continuous decrease in the number of confined segments as the gap width is decreased (Figure 10a).
The calculated free-energy changes upon increased confinement of these systems show oscillatory attractive values for the spherical and straight-chain alkane molecular films and overall repulsive (positive) values for squalane (Figure 11). The energetic and entropic components of the solvation forces were investigated, and it is found that in the spherical and straightchain molecular films they exhibit regular oscillations, while for the branched alkane their variation is irregular and of a more monotonic nature (Figure 12).

These characteristics, which are correlated with the above mentioned structural properties of the confined films, suggest that liquids with molecular structures that are more conducive to formation of ordered configurations (such as globular or lowmolecular weight straight-chain molecules) would develop solidlike characteristics under confinement, ${ }^{50}$ while confined films made of more complex molecular structures (e.g. a moderate degree of branching, as in squalane), where ordering is frustrated due to the molecular structural complexity, would exhibit a higher tendency to behave in a liquid-like manner. We also remark that these different behaviors were found here to be controlled not by increased chain length (that is, spherical molecules, and in particular, $n$-hexadecane and $n$-tetracosane, show similar properties) but rather by molecular complexity (i.e. branching, as in squalane). In this context we remark that indeed solid-like behavior, under the sole effect of confinement (i.e. with no lateral relative shear motion between the confining surfaces), has been observed recently ${ }^{30}$ in SFA experiments for semispherical molecules (OMCTS). These results may assist the molecular design of future thin-film lubricants.

Acknowledgment. This work is supported by the DOE and the AFOSR. Computations were performed on CRAY Computers at the Pittsburgh Supercomputing Center and at the GIT Center for Computational Materials Science.

\section{References and Notes}

(1) For a recent review see: Bhushan, B.; Israelachvili, J. N.; Landman, U. Nature 1995, 374, 607.

(2) Fundamentals of Friction: Macroscopic and Microscopic Processes; Singer, I. L., Pollock, H. M., Eds.; Kluwer: Dordrecht, 1992.

(3) Physics of Sliding Friction; Persson, B. N. J., Tosatti, E., Eds.; Kluwer: Dordrecht, 1996.

(4) Handbook of Micro/Nano Tribology; Bhushan, B., Ed.; CRC Press: Boca Raton, FL, 1995.

(5) See articles in Langmuir 1996, 12(19), 4481-4609.

(6) Rhykerd, C. L.; Schoen, M. S.; Diester, D.; Cushman, J. Nature 1987, 330, 461. See citations 38-41 cited in ref 1 .

(7) Landman, U.; Luedtke, W. D.; Ribarsky, M. W. J. Vac. Sci. Technol. 1989, A7, 2829.

(8) Thompson, P. A.; Robbins, M. O. Science 1990, 250, 792. Robbins, M. O.; Thompson, P. A. Science 1991, 253, 916.

(9) Landman, U.; Luedtke, W. D.; Ringer, E. M. In ref 2, p 463.

(10) Ribarsky, M. W.; Landman, U. J. Chem. Phys. 1992, 97 1937, and references therein.

(11) (a) Landman, U.; Luedtke, W. D.; Ouyang, J.; Xia, T. K. Jpn. J. Appl. Phys. 1993, 32, 1444. (b) Luedtke, W. D.; Landman, U. Comput. Mater. Sci. 1992, 1, 1.

(12) Landman, U.; Luedtke, W. D. MRS Bull. 1993, 17, 36.

(13) Gupta, S.; Koopman, D. C.; Westerman-Clark, G. B.; Bitsanis, I. A. J. Chem. Phys. 1994, 100, 8444.

(14) (a) Wang, Y.; Hill, K.; Harris, J. G. J. Chem. Phys. 1994, 100, 3276. (b) Ouyang, J. Ph.D. Dissertation, Georgia Institute of Technology, 1995. Ouyang, J.; Luedtke, W. D.; Landman, U. Bull. Am. Phys. Soc. 1995, $40(1), 425$.

(15) Landman, U.; Luedtke, W. D.; Gao, J. Langmuir 1996, 12, 4514

(16) Robbins, M.; Smith, E. D. Langmuir 1996, 12, 4543.

(17) Perry M. D.; Harrison, J. A. Langmuir 1996, 12, 4552.

(18) Manias, E.; Hadziioannou, G.; ten Brinke, G. Langmuir 1996, 12, 4587.

(19) Gao, J.; Luedtke, W. D.; Landman, U. Science 1995, 270, 605.

(20) Gao, J.; Luedtke, W. D.; Landman, U. J. Chem. Phys. 1997, 106, 4309.

(21) Israelachvili, J. N. Intermolecular and Surface Forces, 2nd ed.; Academic Press: New York, 1992. 
(22) Davis, H. T. Statistical Mechanics of Phases, Interfaces and Thin Films; VCH Publishers: New York, 1996.

(23) Persson, B. N. J. Sliding Friction; Springer: Berlin, 1997.

(24) Babuena, P. B.; Berry, D.; Gubbins, K. E. J. Phys. Chem. 1993, $97,937$.

(25) Walley, K.; Schweizer, K. S.; Peanasky, J.; Cai, L.; Granick, S. J. Phys. Chem. 1994, 100, 3361.

(26) Urbakh, M.; Daikhin, L.; Klafter, J. J. Chem. Phys. 1995, 103, 10707. Rozman, M. G.; Urbakh, M.; Klafter, J. Phys. Rev. Lett. 1996, 77, 683.

(27) Granick, S. Science 1991, 253, 1374.

(28) Granick, S.; Demirel, A. L.; Cai, L.; Peanasky, J. Isr. J. Chem. $1995,35,75$.

(29) Israelachvili, J. N.; Horn, R. G. J. Chem. Phys. 1981, 35, 1400.

(30) Klein, J.; Kumacheva, E. Science 1995, 269, 816.

(31) Christenson, H. K.; Gruen, D. W. R.; Horn, R. G.; Israelachvili, J. N. J. Chem. Phys. 1987, 87, 1834.

(32) Israelachvili, J. N.; Kott, S. J.; Gee, M.; Witten, T. A. Macromolecules 1989, 22, 4247.

(33) Gee, M. L. and Israelachvili, J. N. J. Chem. Soc., Faraday Trans. 2 1990, 86, 4049.

(34) For simulations of confined alkanes with a single pendant methyl, where force oscillations similar to those found for the corresponding straightchain alkanes were observed; see ref 14.

(35) Israelachvili, J. N. Private communication.

(36) Chan, D. Y. C.; Horn, R. G. J. Chem. Phys. 1985, 83, 5311.

(37) Israelachvili, J. N. J. Colloid. Interface Sci. 1986, 110, 263.

(38) Reiter, G.; Demirel, A. L.; Granick, S. Science 1994, 263, 1741.

(39) van Alsten, J.; Granick, S. Macromolecules 1990, 23, 4856. Granick, S.; Hu, H.-W. Langmuir 1994, 10, 3857. Peanasky, J.; Cai, L.; Kessel, C. R.; Granick, S.; Hu, H.-W. Langmuir 1994, 10, 3874.
(40) Snook, I. K.; van Megen, W. J. J. Chem. Phys. 1980, 72, 2907.

(41) van Megen, W. J.; Snook, I. K. J. Chem. Phys. 1981, 74, 1409.

(42) Parrinello, M.; Rahman, A. J. Appl. Phys. 1981, 52, 7182. In deriving the equations of motion within the Parrinello-Rahman formalism, we take as our Lagrangian dynamical variables the scaled-space coordinates of the fluid particles and the component of the calculation cell along the $x$ direction.

(43) Mondello, M.; Grest, G. S. J. Chem. Phys. 1995, 103, 7156.

(44) For a recent study of unconfined ultrathin films of linear and branched alkanes on a metal substrate using these interaction potentials, see: Balasubramanian, S.; Klein, M. L.; Siepmann, J. I. J. Phys. Chem. 1996, 100, 11960. For a study of confined hexadecane and squalane, see ref 20 .

(45) Xia, T. K.; Ouyang, J.; Ribarsky, M. W.; Landman, U. Phys. Rev. Lett. 1992, 69, 1967.

(46) Schoen, M.; Diestler, D. J.; Cushman, J. H. J. Chem. Phys. 1987, $87,5464$.

(47) Curry, J. E.; Zhang, F.; Cushman, J. H.; Schoen, M.; Diestler, D. J. J. Chem. Phys. 1994, 101, 10824.

(48) Thompson, P. A.; Robbins, M. O. Phys. Rev. A 1990, 41, 6830.

(49) Thompson, P. A.; Grest, G. S.; Robbins, M. O. Phys. Rev. Lett. 1992, 68, 3448 .

(50) In recent simulations we have found that for the spherical molecules the diffusion constant shows oscillatory variations, with minima occurring for well-formed layered configurations. Furthermore, the diffusion constant decreases significantly, and in a rather abrupt manner, for film thicknesses smaller than six layers (Gao, J.; Luedtke, W. D.; Landman, U., to be published). 